\title{
BMJ Open Trends in characteristics and outcomes among US adults hospitalised with COVID-19 throughout 2020: an observational cohort study
}

John H Page, ${ }^{1}$ Ajit A Londhe, ${ }^{1}$ Corinne Brooks, ${ }^{1}$ Jie Zhang, ${ }^{1}$ J Michael Sprafka, ${ }^{1,2}$ Corina Bennett, ${ }^{1}$ Megan Braunlin, ${ }^{1}$ Carolyn A Brown (D) , ${ }^{1}$ Prista Charuworn, ${ }^{3}$ Alvan Cheng, ${ }^{1}$ Karminder Gill, ${ }^{1}$ Fang He, ${ }^{1}$ Junjie Ma (D) , ${ }^{1}$ Jeffrey Petersen, ${ }^{4}$ Olulade Ayodele, ${ }^{5}$ Ying Bao, ${ }^{6}$ Katherine B Carlson, ${ }^{1}$ Shun-Chiao Chang, ${ }^{5}$ Giovanna Devercelli, ${ }^{5,7}$ Michele Jonsson-Funk, ${ }^{8}$ Jenny Jiang, ${ }^{6}$ Hillary A Keenan, ${ }^{5}$ Kaili Ren, ${ }^{5}$ Kimberly A Roehl, ${ }^{1}$ Lynn Sanders, ${ }^{5}$ Luyang Wang, ${ }^{5}$ Zhongyuan Wei, ${ }^{6}$ Qian Xia, ${ }^{6}$ Peter Yu, ${ }^{5}$ Linyun Zhou, ${ }^{5}$ Julia Zhu (1) , ${ }^{6}$ Kathleen Gondek, Cathy W Critchlow, ${ }^{9}$ Brian D Bradbury ${ }^{1}$

To cite: Page JH, Londhe AA, Brooks C, et al. Trends in characteristics and outcomes among US adults hospitalised with COVID-19 throughout 2020: an observational cohort study. BMJ Open 2022;12:e055137. doi:10.1136/ bmjopen-2021-055137

- Prepublication history and additional supplemental material for this paper are available online. To view these files, please visit the journal online (http://dx.doi.org/10.1136/ bmjopen-2021-055137).

Received 13 July 2021 Accepted 02 February 2022

Check for updates

(c) Author(s) (or their employer(s)) 2022. Re-use permitted under CC BY-NC. No commercial re-use. See rights and permissions. Published by BMJ.

For numbered affiliations see end of article.

Correspondence to Dr John H Page; jopage@amgen.com

\section{ABSTRACT}

Objectives To examine the temporal patterns of patient characteristics, treatments used and outcomes associated with COVID-19 in patients who were hospitalised for the disease between January and 15 November 2020.

Design Observational cohort study.

Setting COVID-19 subset of the Optum deidentified electronic health records, including more than 1.8 million patients from across the USA.

Participants There were 51510 hospitalised patients who met the COVID-19 definition, with 37617 in the laboratory positive cohort and 13893 in the clinical cohort.

Primary and secondary outcome measures Incident acute clinical outcomes, including in-hospital all-cause mortality.

Results Respectively, $48 \%$ and $49 \%$ of the laboratory positive and clinical cohorts were women. The 50-65 age group was the median age group for both cohorts. The use of antivirals and dexamethasone increased over time, fivefold and twofold, respectively, while the use of hydroxychloroquine declined by $98 \%$. Among adult patients in the laboratory positive cohort, absolute age/ sex standardised incidence proportion for in-hospital death changed by -0.036 per month $(95 \% \mathrm{Cl}-0.042$ to $-0.031)$ from March to June 2020, but remained fairly flat from June to November, $2020(0.001(95 \% \mathrm{Cl}-0.001$ to 0.003 ), $17.5 \%$ (660 deaths /3986 persons) in March and $10.2 \%$ (580/5137) in 0ctober); in the clinical cohort, the corresponding changes were $-0.024(95 \% \mathrm{Cl}-0.032$ to $-0.015)$ and 0.011 (95\% Cl 0.0070 .014$)$, respectively $(14.8 \%$ (175/1252) in March, 15.3\% (189/1203) in October). Declines in the cumulative incidence of most acute clinical outcomes were observed in the laboratory positive cohort, but not for the clinical cohort.

Conclusion The incidence of adverse clinical outcomes remains high among COVID-19 patients with clinical diagnosis only. Patients with COVID-19 entering the hospital are at elevated risk of adverse outcomes.

\section{Strengths and limitations of this study}

The data represents a wide cross-section of hospitalised patients with COVID-19 across the USA.

- This study highlights the epidemiology of COVID-19 hospitalisations among patients without a positive SARS-CoV-2 RNA/antigen test coincident with their clinical disease.

- The data provided near-real-time availability of relatively rich data on in-hospital interventions, laboratory measurements and all-cause mortality.

- The data may not capture a patient's entire encounter history, including encounters with providers outside the electronic health record network.

- The electronic health records did not include all information on symptoms and orders made in the hospital, limiting the ability to report on the use of some oxygen therapies.

\section{INTRODUCTION}

COVID-19, which causes vascular thrombosis, an acute respiratory distress syndrome (ARDS)-like illness, and vascular and neurological complications, was first reported in Wuhan, China in December 2019, before spreading worldwide to become a pandemic. ${ }^{1}$ Early reports suggest that $5 \%-20 \%$ of patients with COVID-19 developed critical illness characterised by a syndrome similar to ARDS. ${ }^{2-9}$ COVID-19 is theorised to compose of an early viral replication phase followed by an immune dysregulation phase. ${ }^{10}$ It is during the latter phase that many patients are hospitalised.

As of May 2021, the USA had over 32 million COVID-19 cases and over 582000 deaths. ${ }^{11}$ Information on the clinical spectrum of 
severe COVID-19 in the USA has been consistent with that seen in China and Europe. ${ }^{12-20}$ A number of these reports have described the characteristics of patients with COVID-19 presenting between February and April 2020, early in the pandemic.

In the first months of the pandemic, several treatment strategies were tried based on earlier experience with SARS and MERS. As the understanding of the disease evolves, and treatments are evaluated in clinical trials, the most commonly used clinical management strategies are constantly evolving and changing. Some studies involving hydroxychloroquine ${ }^{21-23}$ and lopinavir/ritonavir ${ }^{24-27}$ did not demonstrate clinical benefit as compared with the usual standard of care. By September 2021, several therapies had received regulatory approval or emergency use authorisation including antiviral remdesivir alone, ${ }^{28}$ remdesivir in combination with baricitinib, ${ }^{29}$ dexamethasone, ${ }^{30}$ tocilizumab, ${ }^{31-33}$ convalescent plasma. ${ }^{34}$ Close clinical observation and much trial and error have also resulted in improvements in the management of patients admitted to the intensive care unit (ICU). ${ }^{35} 36$

Other authors have described changes in clinical characteristics and outcomes over time in the USA, and related those changes to changes in therapy or overcrowding. ${ }^{3738}$ To our knowledge, no study to date, has evaluated the relationship between COVID-19 and the availability of SARS-CoV-2 RNA/Ag testing. In this paper, we characterise the clinical course, including clinical manifestations, disease progression, clinical complications and outcomes, of adult patients hospitalised with COVID-19 and explore how these factors differ over time. Importantly, we distinguish between patients with a positive SARS-CoV-2 RNA or antigen test and those with only a clinical diagnosis of COVID-19. We also study how the changes in outcomes varied with the availability of SARSCoV-2 RNA/Ag testing.

\section{METHODS}

\section{Data source}

This cohort study used the COVID-19 subset of the Optum deidentified EHR database. ${ }^{39}$ The database, which is commercially available as a secondary data source, contains longitudinal data from dozens of healthcare provider organisations that include more than 700 hospitals and 7000 clinics. Electronic health record data are sourced from integrated delivery network systems and ambulatory networks from all 50 US states. All payer types are represented, including public (Medicare, Medicaid), commercial, other payor types and uninsured. Data elements include demographics (age, gender, race, ethnicity, census division); medications prescribed and administered; lab results; vital signs and other observable measurements; clinical diagnoses (except symptom data) and procedures; and death events at a month level, however, oxygen therapy was not reliably recorded in this database. There is higher patient representation in this database from the East North Central, South Atlantic and West South Central US Census divisions.

Sourced from EHR data, the Optum COVID-19 database may not capture the entirety of patients' encounter history among all healthcare systems. The Optum COVID-19 database ${ }^{40}$ is certified as deidentified by an independent statistical expert following HIPAA statistical deidentification rules, and was exempt of IRB approval. As such, certain data elements, such as death events and patient location, are available at imprecise levels to reduce the risk of patient identifiability.

The COVID-19 subset of the Optum database used in this study includes more than 1.8 million patients, updated and refreshed up to 2 weeks prior to delivery. The end date of the study database was 27 January 2021. For inclusion into the COVID-19 database, patients must have a clinical encounter related to COVID-19 (eg, clinical diagnosis or lab test).

Algorithms to define clinical events were developed to mitigate database limitations. For example, dates on which patients were known to be physically present and alive through diagnosis and procedure clinical codes were used to infer a more precise death date from the available death month information. This same process was implemented to obtain more specific durations of healthcare utilisation (eg, hospitalisation dates and ICU). All algorithms used to provide more precision in clinical event identification are described in online supplemental algorithms 1-4.

\section{Cohort}

The study population includes patients 18 years and older who were hospitalised with COVID-19 clinical diagnoses and/or positive SARS-CoV-2 tests. Because we have previously calculated a 6-week lag in death date completeness in the data ${ }^{41}$ and required at least 4 weeks of observation for outcomes, we required COVID-19 diagnoses no later than 10 weeks before the database end date, which is 15 November 2020. Therefore, a COVID-19 diagnosis was defined as the first occurrence of any of the following (1) ICD-10-CM code B97.29 or B34.20 occurring on or before 30 April 2020; (2) positive SARS-CoV-2 RNA or antigen test between 1 February 2020 and 15 November 2020; (3) ICD-10-CM diagnosis code U07.1, J12.81, J12.89 or J80 between 1 February 2020 and 15 November 2020. Patients needed to be hospitalised on or within 28 days after the COVID-19 diagnosis date (online supplemental figure 1).

Patients were then assigned one of two cohorts: patients with a positive viral RNA/antigen test (SARS-CoV-2 positive, referred to as the laboratory positive cohort); or patients without a positive viral RNA/antigen test within 21 days of the qualifying diagnosis but were included due to one of the diagnosis codes (clinical diagnosis only, referred to as the clinical cohort). The clinical cohort consisted of patients with either no documented SARSCoV-2 RNA/antigen test, a negative test, or a positive test of an unknown test type. 
Patients were excluded for any of the following reasons: (1) missing age or gender data; (2) continuous hospitalisation for more than 10 days before the first COVID-19 diagnosis; (3) diagnosis or procedure codes for labour and delivery during hospitalisation or (4) diagnosis codes for trauma, injury, fracture or poisoning during the first 2 days of hospitalisation. Among patients with multiple hospitalisations that met the study criteria, only the earliest hospitalisation was included in this analysis. Hospitalised patients may not be included in the data if their admitting facility is outside the EHR network. There is no minimum requirement for the length of baseline period in the database prior to index date.

\section{Index date}

For baseline characteristic and treatment analyses, the index date was defined as the earlier of diagnosis date or hospital admission date; follow-up started on index date and ended at the earlier of death date, hospital discharge, or database end date.

For outcome analyses, the index date was defined as the hospital admission date; follow-up started on index date and ended at the earlier of death date, hospital discharge or 28 days after hospital admission.

\section{Variables of interest and outcomes}

Variables of interest included baseline demographics and comorbidities ascertained between 30 days and 12 months prior to index date; comedications within 12 months prior to index date; outcomes within hospitalisation and medications administered from index date to end of hospitalisation. We chose to examine medications and supplements that were either widely used by clinicians $^{42}$ or were being evaluated in clinical trials ${ }^{43}$ and had sufficient use in the data. Outcomes included all-cause mortality; receipt of medications or procedures indicating severe disease course (ICU admission, extracorporeal membrane oxygenation (ECMO), mechanical ventilation, vasopressor usage); indicators of organ-specific manifestations (ARDS or myocarditis) and incident acute clinical outcomes potentially associated with COVID-19 (heart or respiratory failure). Outcome-specific exclusions were applied for chronic conditions to ensure that only incident outcomes were captured. All study outcomes were ascertained and assessed individually. We also examined the distribution of SARS-CoV-2 RNA/antigen test results (not tested vs negative) in the clinical cohort by index date month as well as the top 20 discharge codes observed in the laboratory positive cohort by month.

\section{Data analysis}

Continuous outcomes were presented as means, and categorical outcomes were described with patient numbers, percentages and proportions. Cumulative incidence of study outcomes was plotted (1) over time and stratified by age group and (2) by month (after standardisation for the age/sex distribution in the entire hospitalised cohort: performed by modelling the month/laboratory positive versus clinical interaction as outcome in a multinomial logistic regression and using the reciprocal of the respective predicted probabilities as weights in the models for cumulative incidence). Cumulative incidence was calculated as the number of events in the follow-up period divided by the total number of patients in the cohort. Patients were censored at the database end date. Date of discharge was treated as a competing risk in estimates of cumulative incidence of all-cause mortality, whereas dates of discharge and death were treated as competing risks in estimates of cumulative incidence for the other studied outcomes, using the method of Fine and Gray. ${ }^{44}{ }^{45}$ To evaluate the relationship between cumulative incidence of death and month, we first plotted the cumulative incidence in each of the two cohorts (laboratory positive and clinical cohorts) by month. After examining the plot, we identified a breakpoint and used that as the basis for generating linear splines. We then estimated a linear regression model with all-cause mortality as an outcome, and period-specific linear spline, cohort and the periodspecific linear spline by cohort interaction as predictors, weighted by the reciprocal of the probability that the patient's respective age group-sex is a part of the study sample as a whole.

To inspect baseline trends over time, patients were stratified by season of index date (January-May, June-August, September-15 November). The seasonal breakdown corresponds with the three US surges in COVID-19 transmission: spring, summer and autumn. Patients were stratified by month of index date and by age group (18-34, $35-49,50-64,65-74,75-84,85+)$ in the assessment of outcomes.

Finally, to explore how changes over time in the weekly proportion of hospitalised patients tested for SARS-CoV-2 could affect all-cause mortality trends, we estimated the former among hospitalised patients in the primary Optum cohort, standardised by the age/sex distribution in the hospitalised COVID-19 cohort over the duration of the study. The positivity proportion was based on patients' RNA/antigen testing status during the time beginning 28 days prior to hospital admission and ending on the earlier of hospital discharge or 10 days postadmission.

Data processing and characterisation were performed using SAS V.9.4 (SAS Institute) and R V.4.0.3 (R Core Team, 2020).

\section{Patient and public involvement}

No patient or public involvement in the study as deidentified patient dataset was used in the analysis.

\section{RESULTS}

Of the 585867 patients who met the COVID-19 definition during the study period, 333967 (57\%) were adults with at least 10 weeks between the index date and the last database refresh date (online supplemental figure 2). Of these, 61693 were hospitalised, and 51510 (83\%) of the hospitalised patients met the inclusion criteria, 
comprising the study population with $37617(73 \%)$ in the laboratory positive cohort and 13893 (27\%) in the clinical cohort. Most patients were from the East North Central and Middle Atlantic divisions in January-May, the East North Central and South Atlantic divisions in June-August and the East North Central and West North Central divisions in September-November (table 1, baseline medication usage is available in online supplemental tables 1 and 2). The Mountain and Pacific divisions were under-represented in the data. The proportion of Caucasians increased over time (laboratory positive: $48 \%, 58 \%$ and $73 \%$, clinical: $53 \%, 64 \%$ and $73 \%$ ), whereas the proportions of African Americans (laboratory positive: $28 \%, 23 \%$ and $12 \%$, clinical: $26 \%, 18 \%$ and $13 \%$ ) and Asians (laboratory positive: $4 \%, 2 \%$ and $1 \%$, clinical: $2 \%$, $2 \%$ and $1 \%$ ) decreased. The proportion of patients 18-34 years of age increased between January-May (laboratory positive: $6.7 \%$, clinical: $8.2 \%$ ) and June-August (laboratory positive: $10.5 \%$, clinical: $9.9 \%$ ) before falling in September-November (laboratory positive: $6.9 \%$, clinical: $6.7 \%$ ). The proportion of the clinical cohort without a documented SARS-CoV-2 RNA/Ag test varied with time, $100 \%$ in February, $83.1 \%$ in March, $82.5 \%$ in April, falling to $64.0 \%$ in June, steadily rising to $81.1 \%$ in November 2020 (online supplemental figure 3).

Of the therapies examined, the most common administered included analgesics/antipyretics, antibiotics, anticoagulants and bronchodilators (figures 1 and 2 and online supplemental table 3). Over a quarter of patients in the laboratory positive cohort received nutritional supplementation in each time, with vitamin $\mathrm{D}$ being the most frequently administered. Early use of antibiotics was observed in the laboratory positive cohort $(78 \%$ in January-May) and the clinical cohort (68\%). Remdesivir in the laboratory positive cohort increased from $4 \%$ in January-May to $45 \%$ in September-November, and the clinical cohort increased from $3 \%$ to $27 \%$. In both cohorts, hydroxychloroquine use decreased from January-May (laboratory positive 51\%; clinical cohort $15 \%)$ to less than $1 \%$ by September-November. The use of systemic steroids (particularly dexamethasone) was greater than $60 \%$ in the laboratory positive cohort in all time frames except January-May. There was increased use of convalescent plasma over time $(0.02 \%$ in January-May and $16 \%$ in September-November in the laboratory positive cohort), although medical codes for convalescent plasma only became available in August 2020.

The cumulative risk of all-cause mortality, standardised by age and sex within 28 days of index date (figure 3), in the laboratory positive cohort decreased from $17.5 \%$ in March to $15.7 \%$ in April and $11.3 \%$ in May, and then remained between $9.6 \%$ and $10.8 \%$ for the remainder of the study. The regression analysis of all-cause mortality trends in this cohort revealed that absolute age/sex standardised incidence proportion for in-hospital death changed by -0.036 per month $(95 \%$ CI -0.042 to -0.031 ) 0.001 ( $95 \%$ CI -0.001 to 0.003 ) from March to June 2020, but was 0.001 (95\% CI -0.001 to 0.003 ) from June to November 2020 (online supplemental figure 4). The clinical cohort had a mortality cumulative risk of $14.8 \%$ in March; the risk lowered to $8.9 \%-12.5 \%$ in May through August, then rose again to $16 \%$ in September, before dropping to $15.3 \%$ in October and $11.8 \%$ in November. The regression analysis of mortality trends in this cohort revealed that absolute age/sex standardised incidence proportion for in-hospital death changed -0.024 per month $(95 \%$ CI -0.032 to -0.015$)$ from March to June 2020, but was 0.011 (95\% CI 0.007 to 0.014 ) from June to November 2020 (online supplemental figure 4). The pattern of mortality seen in the clinical cohort was fairly similar to the pattern of mortality for the subset of the cohort without a documented SARS-CoV-2 RNA/Ag test, and different from those patients with a documented negative SARS-CoV-2 RNA/Ag (online supplemental figure 5). Additional information on cumulative risks for all outcomes can be found in online supplemental table 4 and online supplemental figures 6-8.

In the laboratory positive cohort, the age-standardised and sex-standardised cumulative incidence of ARDS decreased from 19\% in March to 6\% in August, then remained between $5 \%$ and $6 \%$ in September through mid-November. The clinical cohort had a higher agestandardised and sex-standardised cumulative incidence of ARDS in March (26\%) and a lower incidence in April $(11 \%)$ and May (10\%). An increased cumulative incidence of ARDS was observed after June in the clinical cohort. Similar to ARDS, the cumulative incidence across several other outcomes declined before September and became stable after September in the laboratory positive cohort, including acute respiratory failure, ECMO/invasive mechanical ventilation use, arrhythmia, myocarditis, venous thromboembolism, ICU admission and acute heart failure. In the clinical cohort, similar declines were observed before June, and increases in cumulative incidence were observed after June for most outcomes (online supplemental figure 6). The proportion of patients admitted to the ICU on the day of admission varied with period (respectively, $10.8 \%, 6.5 \%$ and $6.6 \%$ in January to May, June to August and September to November for the laboratory positive cohort, and $14.2 \%$, $11.2 \%$ and $12.1 \%$ for the corresponding periods in the clinical cohort).

The rise in the proportion of hospitalised patients who were tested among the cohort of hospitalised patients from the primary Optum EHR database increased from $3 \%$ to $61 \%$, which mirrors the drop in all-cause mortality among the laboratory positive cohort (figure 4). The positivity proportion, which began at $11 \%$, peaked in late March at 36\% before dropping over the same timeframe to $4 \%$. Assessing the most common discharge codes observed in the laboratory positive cohort, the proportion of several diagnosis codes related to COVID-19, including sepsis and pneumonia, decreased over time, whereas a code for atherosclerotic heart disease increased over the same time (data not shown). The proportion of patients with discharge code of COVID-19 (U07.1) varied between 
Table 1 Baseline characteristics of hospitalised patients with COVID-19 by cohort and period

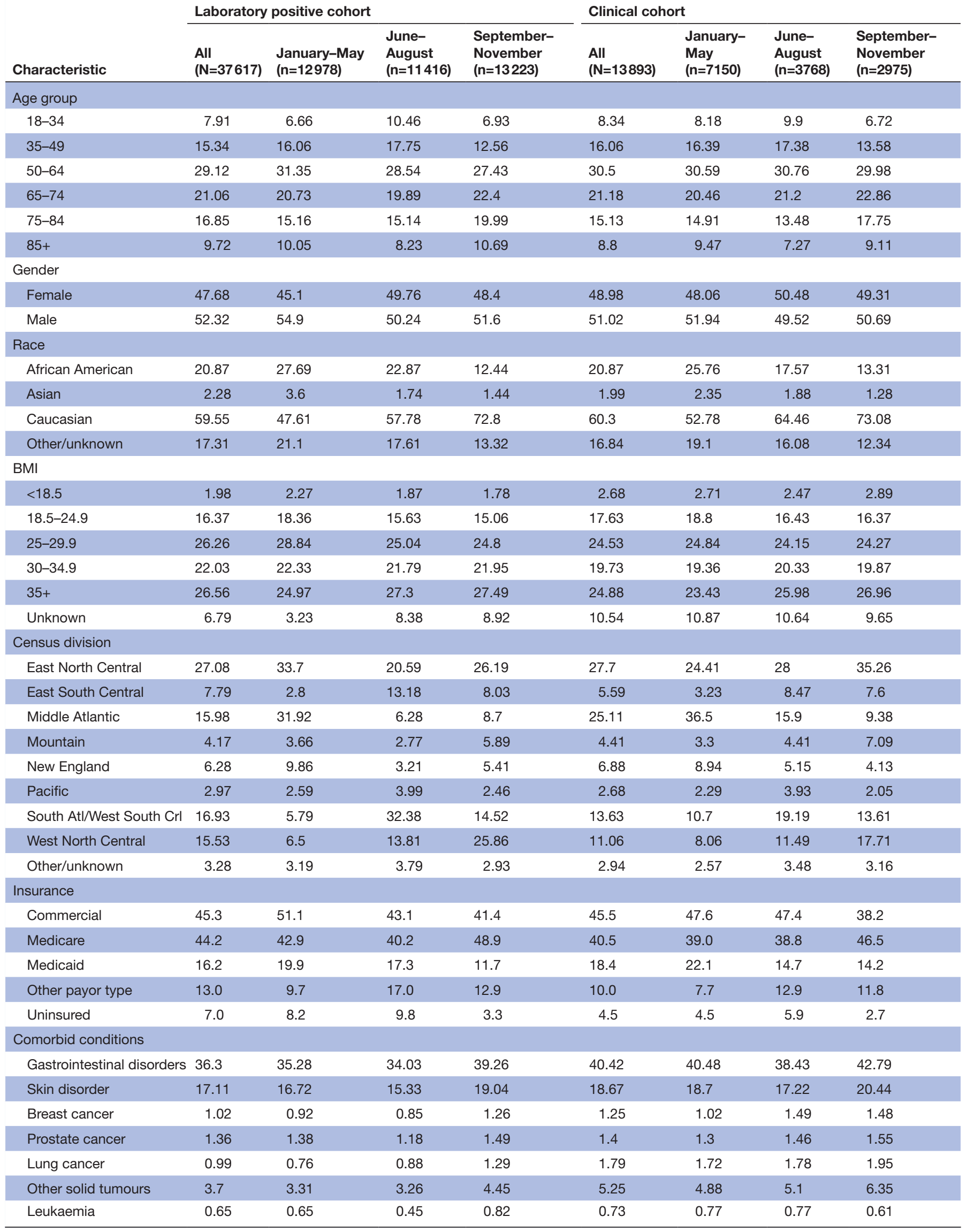


Table 1 Continued

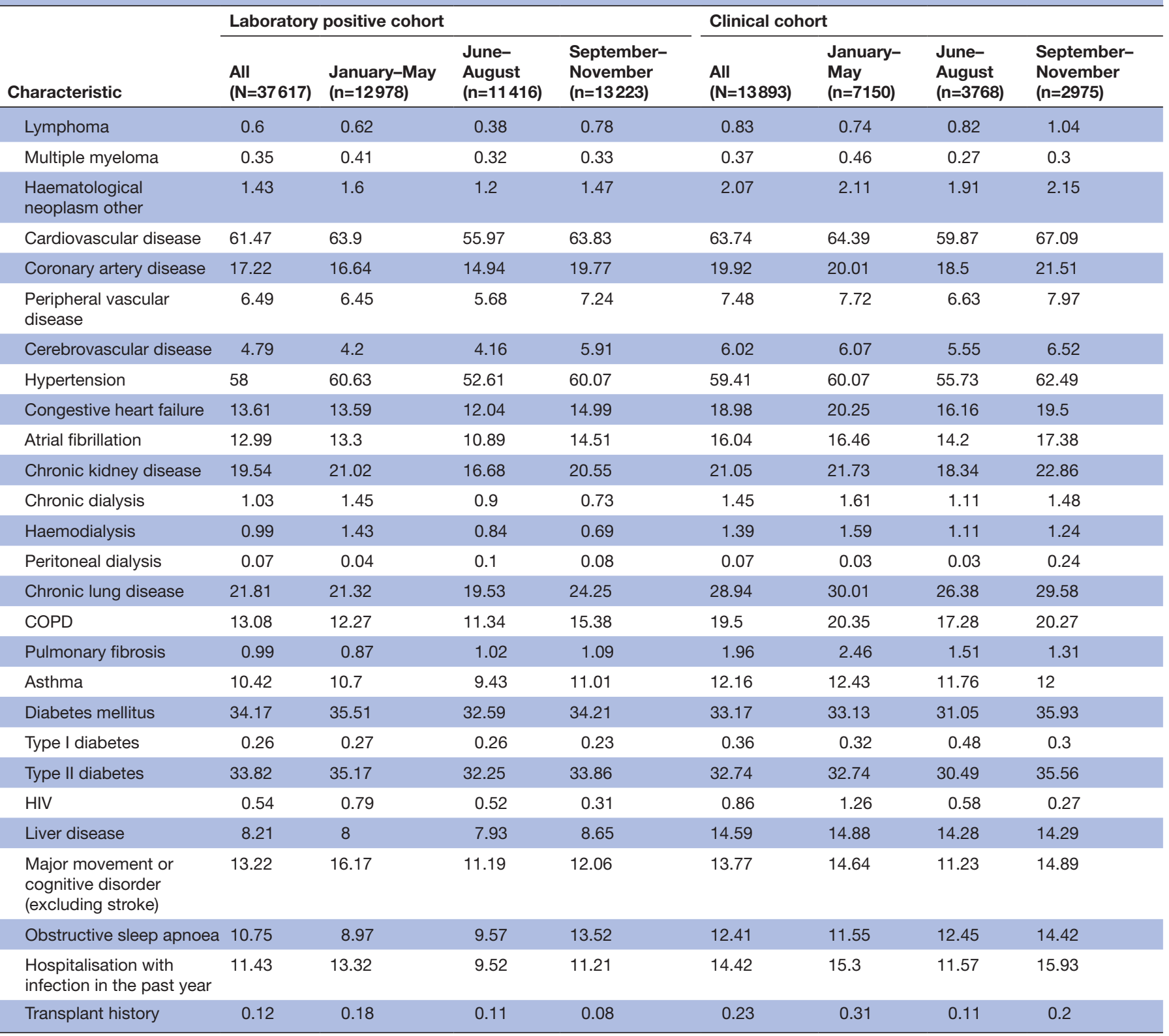

Proportions of baseline characteristics among SARS-CoV-2 positive (laboratory positive) and clinical diagnosis only (clinical) cohort patients stratified by time periods. All values are column percentages. Insurance is summarised from the last, non-null clinical encounter during baseline to 7 days after index date.

$64.7 \%$ and $76.4 \%$ from May to November, where discharge codes were available ( $18.1 \%$ of the study cohort).

\section{DISCUSSION}

In the USA, over the first 10 months of the COVID-19 pandemic, we saw an average decline of $52 \%$ (range: $38 \%-74 \%)$ in the age-standardised and sex-standardised incidence of adverse events of COVID-19 in the laboratory positive cohort that coincided with the increase in widespread availability of SARS-CoV-2 RNA/antigen testing in the USA, and with the increasing temperatures associated with the change in seasons. We also saw a decline in the age-standardised and sex-standardised incidence of adverse events of COVID-19 in the clinical cohort that coincided with summer, but unlike the laboratory positive cohort, the incidence in adverse events increased in the autumn. The changes seen in the laboratory positive cohort are consistent with other recent reports in the medical literature. ${ }^{46-49}$

Others have speculated about reasons for the changes in the cumulative incidence of in-hospital adverse outcomes of confirmed COVID-19 admissions, including changes in treatment pattern, ${ }^{28} 30375051$ crowding, ${ }^{38} 52$ changes in viral characteristics ${ }^{53}$ and the implementation of non-pharmaceutical interventions (physical distancing, decreased indoor gathering and the use of facemasks) in many regions of the USA, ${ }^{54-56}$ beginning in April 2020. However, few researchers have accounted for the 


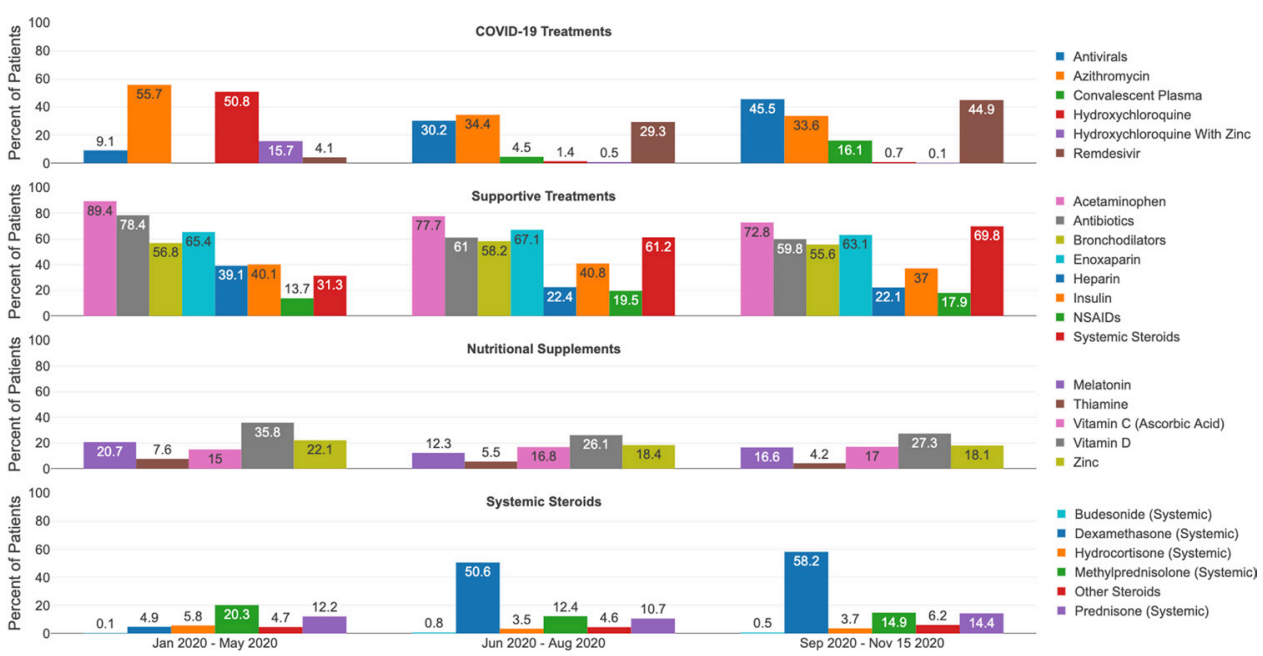

Figure 1 COVID-19 treatments in laboratory positive cohort, per period.

changing availability and use of SARS-CoV-2 testing throughout 2020. Testing was not available before March 2020 and there was increasingly frequent use from May onward. This widespread use was coincident with a marked decline in the proportion of patients testing positive (figure 4). In addition, many hospitals started testing all or most of their inpatients for SARS-CoV-2 beginning in late April to May. ${ }^{57}$ This may have falsely inflated the denominators, resulting in a consequent fall in event incidence rates. This inflation of the denominator of the laboratory positive cohort is less likely to have occurred in the clinical cohort, among whom the majority of patients either did not have a documented SARS-CoV-2 RNA/ antigen test result or had a documented negative SARSCoV-2 RNA/antigen test result.

Further, the decline in adverse events did not coincide with increased use of therapies known to be effective in reducing mortality. ${ }^{30}{ }^{58}$ The first publication of dexamethasone randomised clinical trial was announced in the press on June $2020^{59}$ and was published in October $2020 .^{58}$ In our data, dexamethasone use was seen in over $10 \%$ of hospitalised patients for the first time in June
2020, and over $50 \%$ in July 2020. Prevalence of systemic steroid use was over $50 \%$ in January and February, before falling to 25\%-33\% between March and May, then rising to over $40 \%$ in June and was $~ 60 \%$ in July 2020 .

We suspect that much of the decline in adverse effects among the laboratory positive cohort was due to disease misclassification, in that, although infected with SARS-CoV-2 virus, many of these patients likely did not have disease due to SARS-CoV-2 infection (COVID-19 warranting hospitalisation) and among these patients, we observed declines in the proportion of patients with discharge codes for severe illnesses. This is somewhat counterintuitive because this cohort all had positive tests for SARS-CoV-2. However, many patients with SARS-CoV-2 infection are either asymptomatic or have mild symptoms ${ }^{60}$ and a number of these patients would still need hospital admission for reasons unrelated to their SARSCoV-2 infection. Although we attempted to address this by excluding patients admitted with trauma or obstetric conditions, we likely did not exclude all patients who should have been excluded from the cohort. The fall in the proportion of these patients admitted to the ICU

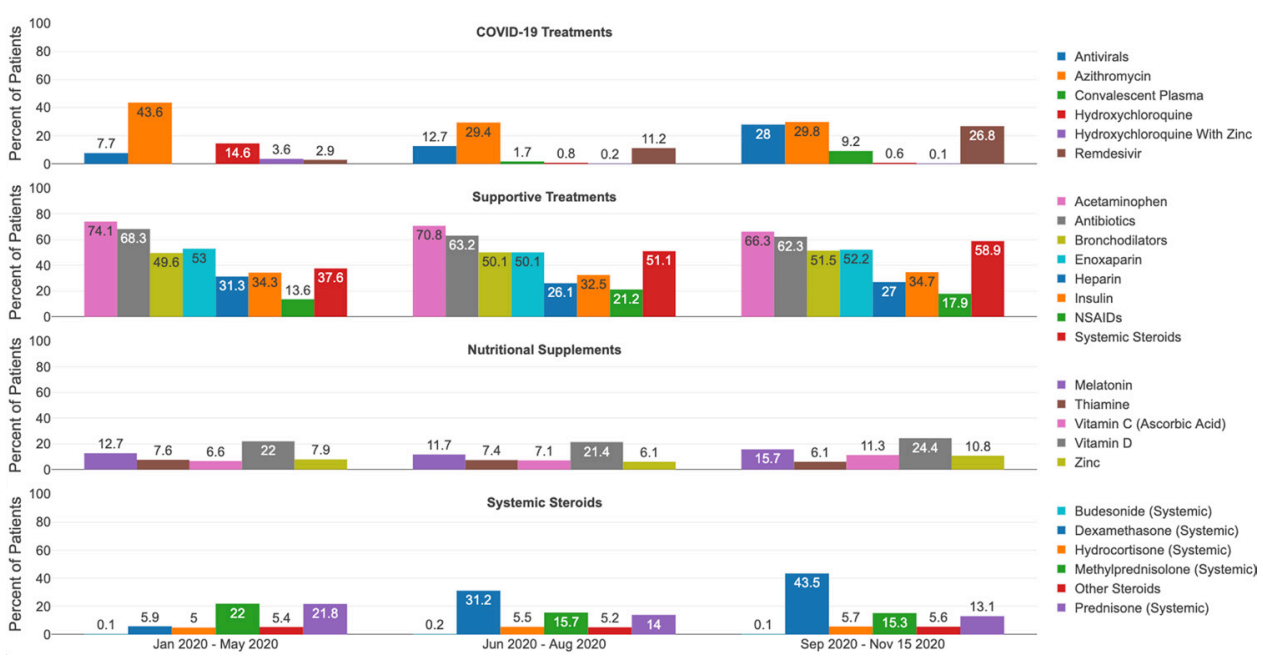

Figure 2 COVID-19 treatments in clinical cohort, per period. 

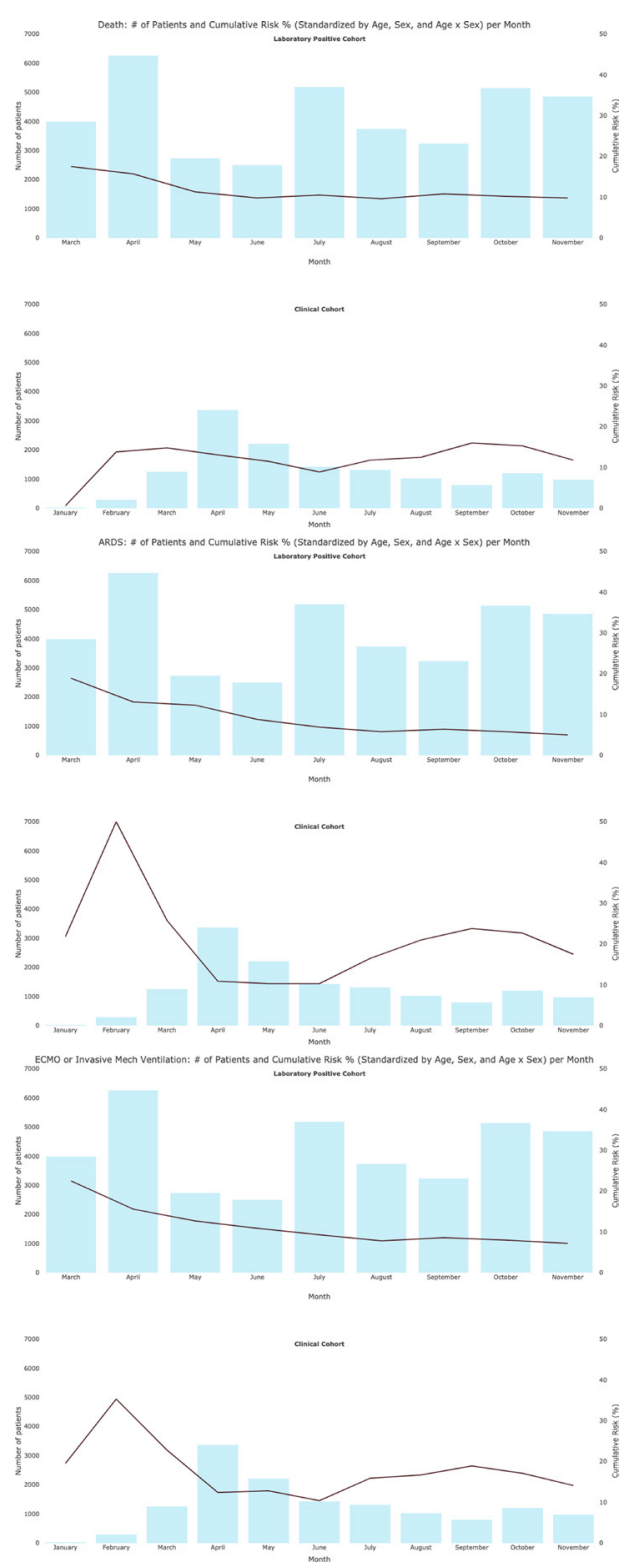

Figure 3 Patient counts and cumulative risk of select outcomes by month, standardised by age and sex.

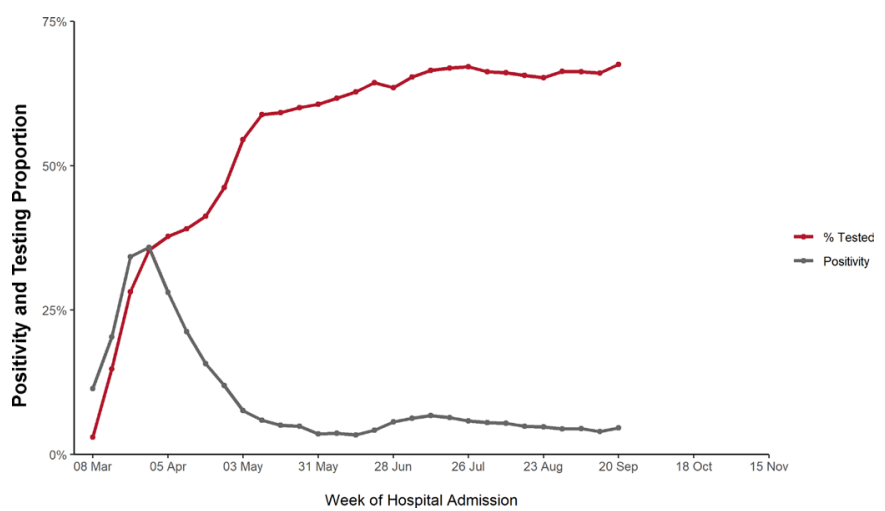

Figure 4 Positivity and testing proportion, standardised by age and sex. and/or treated with invasive mechanical ventilation on their day of admission supports this hypothesis.

With respect to test results, the clinical cohort was primarily composed of two groups: (1) patients with at least one negative SARS-CoV-2 RNA/antigen test either in the 28 days prior to hospital admission or during the first 10 days of admission, but no positive test in the period; (2) no documented SARS-CoV-2 RNA/antigen test result in the 28 days prior to hospital admission or during the first 10 days of admission. There were also some patients who had a positive SARS-CoV-2 related test result of an unknown test type. Compared with the laboratory positive cohort, while most baseline characteristics were similar, the percentage of patients with certain comorbid conditions in the clinical cohort was higher, and differences were observed with respect to census division. Further, the patients in the clinical cohort were more likely to be admitted to the ICU or treated with invasive mechanical ventilation on the day of admission. It is possible that some of these patients did not have COVID$19,{ }^{61}$ but we know that SARS-CoV-2 testing is associated with false negatives, ${ }^{62} 63$ and it is clear that many of the patients in this cohort were quite ill. It should also be noted that patients are more likely to have virus shedding in the early symptomatic phase than in the later immune dysregulation phase when patients are more likely to hospitalised. ${ }^{1064}$ Although the SARS-CoV-2 RNA PCR may remain positive after virus shedding has ceased, there is a correlation. ${ }^{64}$ Earlier research has suggested that due to inaccurate testing for COVID-19, a patient suffering from COVID-19 symptoms coupled with a recent exposure should be diagnosed clinically even in the context of a negative test. ${ }^{65}$ Further, some investigators were able to detect SARS-CoV-2 RNA on bronchoalveolar lavage in up to $16 \%$ of patients negative with nasopharyngeal testing. ${ }^{6}{ }^{6}$ The pattern of mortality seen in the clinical cohort seemed to have been primarily driven by the mortality patterns in the group of patients without a documented SARS-CoV-2 RNA/Ag test. Although testing was largely unavailable before mid-March when the pandemic was still in its early phase, the same cannot be said for later in the year, particularly after May 2020. We therefore caution comparing outcomes after April 2020 with those before then, for the clinical cohort. It is possible that a subset of these patients was transferred from other facilities (with missing documentation) and thus have worse prognosis when compared with other patients. It is also possible that some patients were not tested because they arrived at hospital already intubated. Given the lower proportion of patients with Medicaid and/or uninsured in autumn compared with summer, we do not think that worse outcomes later in the year was due to patients coming from lower socioeconomic settings.

The strengths of this research include the study size, geographical coverage, large size of dataset and longitudinal nature. These data represent a wide cross-section of hospitalised patients with COVID-19 over the initial 10 months of the COVID-19 pandemic across the USA, 
with a diverse mix of insurance types, socioeconomic status and demographic factors. The provided data were relatively rich regarding in-hospital interventions and laboratory measurements. The availability of in-hospital mortality data and the near-real-time availability of the patient EHRs were advantages of this study.

\section{Limitations}

The Optum COVID-19 EHR database may not capture a patient's entire encounter history in the healthcare system. Some patients who have outpatient events may have hospitalisations that do not appear in the data if the admitting facility is not included in the EHR network. Conversely, hospitalised patients with data available may have outpatient events that do not appear if those facilities were outside of the EHR network. This limitation likely impacted our assessment of baseline characteristics and therapies, including the apparently large proportion of patients who were hospitalised (online supplemental figure 2). Further, the data did not provide information on all symptoms, and not all patients had discharge codes available, as only a subset of provider networks provide this information. We were unable to ascertain the reasons for which medications used were prescribed, and therefore it may be possible that some therapies may not have been prescribed to treat COVID-19. Detailed information on all orders made in hospital were not available to the study team, and thus we were not able to reliably report on the use of some oxygen therapies during hospitalisation, nor were we able to evaluate some ICU management strategies, such as placing patients in the prone position to decrease the need for invasive ventilation. With no ICD-10-CM code specified for COVID-19 available until 30 April 2020 and inconsistent usage of it after its inception, we used a set of proxy ICD-10-CM codes indicated for COVID-19 diagnosis early in the pandemic to ensure sensitivity throughout 2020. The use of these proxy diagnosis codes likely reduced specificity of COVID-19 status in the clinical cohort. Race/ethnicity characterisation in the database is not well captured and prior studies have identified shortcomings. ${ }^{67}$ In the laboratory positive cohort, lab names and result values for SARS-CoV-2 testing were not available in a standardised format, so a clinical review process was needed to ascertain the test type and result. There was a significant lag in the recording of several outcome events, which led us to restrict the examination of the cohort to index dates prior to 15 November 2020 , which is minimally 10 -week follow-up from database release date of 27 January 2021. It is important to note that we did not evaluate the effectiveness of the therapies used with respect to patient outcomes.

\section{CONCLUSIONS}

To our knowledge, this is the first study to highlight the epidemiology of COVID-19 hospitalisations among patients without a positive SARS-CoV-2 RNA/antigen test coincident with their clinical disease. We believe that these patients have not received the attention that they deserve, and although we believe that there is some misclassification, it is important that this topic is a focus for future research. To our knowledge, it is also the first study to highlight the likely impact of the inflation of the denominators with patients infected with SARS-CoV-2, but without COVID-19 warranting hospitalisation. These findings have important implications, not just for researchers studying COVID-19, but also for clinicians. COVID-19 remains a major threat, and consistent with the phase of the illness, it is advisable that clinicians promptly treat COVID-19 patients, with or without a corresponding positive SARS-CoV-2 RNA/antigen test. The need to improve the management and availability of effective therapies for COVID-19 remains a high priority. The findings from this study are based on data from the first 10 months of the COVID-19 pandemic in the USA. While these findings may not be directly generalisable to the future of this pandemic, they may have implications for any situation where the criteria for entry into the cohort changes over time. Future studies should further evaluate the nature of disease in hospitalised patients with COVID-19 who are negative on SARS-CoV-2 RNA/antigen testing and the reasons some patients do not have documentation of testing. In addition, there should be attempts to distinguish between patients admitted because of COVID-19 and patients admitted for other reasons but with a positive SARS-CoV-2 test.

\section{Author affiliations}

${ }^{1}$ Center for Observational Research, Amgen Inc, Thousand Oaks, California, USA

${ }^{2}$ Woodford Research Associates, Thousand Oaks, California, USA

${ }^{3}$ Inflammation, Global Development, Amgen Inc, Thousand Oaks, California, USA

${ }^{4}$ Medical Sciences, Amgen Inc, Thousand Oaks, California, USA

${ }^{5}$ Data Sciences Institute, Takeda Pharmaceutical Company Limited, Cambridge, Massachusetts, USA

${ }^{6}$ Center for Observational Research and Data Science, Bristol-Myers Squibb, Princeton, New Jersey, USA

${ }^{7}$ Global Evidence and Outcomes, Takeda Pharmaceutical Company Limited, Pinehurst, North Carolina, USA

${ }^{8}$ Epidemiology, UNC Gillings School of Global Public Health, Chapel Hill, North Carolina, USA

${ }^{9}$ R\&D Human Data, Amgen Inc, Thousand Oaks, California, USA

Acknowledgements We would like to acknowledge the extensive programming and planning work of the Amgen Centre for Observational Research (Oana Abrahamian, Kruti Doshi, Jan Lethen, Yanmei Lu, Hyrum Nielsen, Carrie Nielson, Jessica Spencer and Andrew Toler) and the R\&D Consortium: Takeda (Sudhakar Manne and Vivek Gandhi) and Bristol-Myers Squibb (David Anstatt and Marisa Co). We also would like to acknowledge the healthcare professionals whose tireless efforts in this unprecedented pandemic have provided critical knowledge, as well as the patients from whom we continue to learn so much.

Contributors JHP, AAL, CBrooks, JZ (Amgen) and JMS are guarantors. JHP, AAL, CBrooks, JZ (Amgen), JMS, CAB, AC, FH and JM: study concept and design. JHP, AAL, CBrooks, CWC and BDB: acquisition of data. JHP, AAL, CBrooks, JZ, $\mathrm{CAB}, \mathrm{AC}$, FH and JM: data analysis. JHP, AAL, CBrooks, JZ (Amgen), JMS, CAB, $A C, F H$ and $J M$ : interpretation of data and drafting of manuscript. CBennett, $M B$, $P C, K G, J P, O A, Y B, K B C, S-C C, G D, M J-F, J J, H A K, K R, K A R, L S$, LW, ZW, QX, PY, LZ, JZ (BMS), KG, CWC and BDB: critical revision of manuscript. All coauthors contributed to writing the manuscript. All authors approved the final version of the manuscript.

Funding This study was funded by Amgen Inc., Takeda Pharmaceutical Company Limited and Bristol-Myers Squibb Co. 
Competing interests JHP, AAL, CBrooks, JZ, CBennett, BDB, MB, CAB, PC, AC, CWC, KG, FH, JM, JP and KAR are employees and stockholders of Amgen. JMS reports consulting for Amgen and owns stock in Amgen. OA, S-CC, GD, KG, HK, $\mathrm{KR}, \mathrm{LS}, \mathrm{LW}, \mathrm{PY}$ and LZ are employees and stockholders in Takeda, Pharmaceutical Company Limited. YB, JJ, QX, ZW and JZ are employees and stockholders in Bristol-Myers Squibb. KBC, an employee of Moderna, was formerly an employee of Amgen and owns stock in Amgen. GlaxoSmithKline (GSK), Takeda, AbbVie, Boehringer Ingelheim and UCB Bioscience (UCB) have collaborative agreements with the Center for Pharmacoepidemiology, Department of Epidemiology, University of North Carolina at Chapel Hill which provides salary support to MJ-F and MJ-F is a member of the Scientific Steering Committee (SSC) for a postapproval safety study funded by GSK. All compensation for services provided on the SSC is invoiced by and paid to UNC Chapel Hill.

Patient consent for publication Not applicable.

Ethics approval This study does not involve human participants.

Provenance and peer review Not commissioned; externally peer reviewed.

Data availability statement Data may be obtained from a third party and are not publicly available. The data were used under license for this study with restrictions that do not allow for the data to be redistributed or made publicly available. № additional data are available.

Supplemental material This content has been supplied by the author(s). It has not been vetted by BMJ Publishing Group Limited (BMJ) and may not have been peer-reviewed. Any opinions or recommendations discussed are solely those of the author(s) and are not endorsed by BMJ. BMJ disclaims all liability and responsibility arising from any reliance placed on the content. Where the content includes any translated material, BMJ does not warrant the accuracy and reliability of the translations (including but not limited to local regulations, clinical guidelines, terminology, drug names and drug dosages), and is not responsible for any error and/or omissions arising from translation and adaptation or otherwise.

Open access This is an open access article distributed in accordance with the Creative Commons Attribution Non Commercial (CC BY-NC 4.0) license, which permits others to distribute, remix, adapt, build upon this work non-commercially, and license their derivative works on different terms, provided the original work is properly cited, appropriate credit is given, any changes made indicated, and the use is non-commercial. See: http://creativecommons.org/licenses/by-nc/4.0/.

\section{ORCID iDs}

Carolyn A Brown http://orcid.org/0000-0002-9421-0185

Junjie Ma http://orcid.org/0000-0002-6249-876X

Julia Zhu http://orcid.org/0000-0001-8210-8389

\section{REFERENCES}

1 World Health Organization. Coronavirus disease 2019 (COVID-19): situation report, 72, 2020.

2 Wu Z, McGoogan JM. Characteristics of and Important Lessons From the Coronavirus Disease 2019 (COVID-19) Outbreak in China: Summary of a Report of 72314 Cases From the Chinese Center for Disease Control and Prevention. JAMA 2020;323:1239-42.

3 Guan W-J, Ni Z-Y, Hu Y, et al. Clinical characteristics of coronavirus disease 2019 in China. N Engl J Med 2020;382:1708-20.

4 Grasselli G, Pesenti A, Cecconi M. Critical care utilization for the COVID-19 outbreak in Lombardy, Italy: early experience and forecast during an emergency response. JAMA 2020;323:1545.

5 Yang X, Yu Y, Xu J, et al. Clinical course and outcomes of critically ill patients with SARS-CoV-2 pneumonia in Wuhan, China: a singlecentered, retrospective, observational study. Lancet Respir Med 2020;8:475-81.

6 Zhou F, Yu T, Du R, et al. Clinical course and risk factors for mortality of adult inpatients with COVID-19 in Wuhan, China: a retrospective cohort study. Lancet 2020;395:1054-62.

7 Grasselli G, Zangrillo A, Zanella A, et al. Baseline characteristics and outcomes of 1591 patients infected with SARS-CoV-2 admitted to ICUs of the Lombardy region, Italy. JAMA 2020;323:1574-81.

8 Covino M, De Matteis G, Santoro M, et al. Clinical characteristics and prognostic factors in COVID-19 patients aged $\geq 80$ years. Geriatr Gerontol Int 2020;20:704-8.

9 Ciceri F, Castagna A, Rovere-Querini P, et al. Early predictors of clinical outcomes of COVID-19 outbreak in Milan, Italy. Clin Immunol 2020;217:108509.

10 Marik PE, Iglesias J, Varon J, et al. A scoping review of the pathophysiology of COVID-19. Int J Immunopathol Pharmacol 2021;35:20587384211048026.
11 CDC. Covid data Tracker, 2020. Available: https://covid.cdc.gov/ covid-data-tracker [Accessed 04 Oct 2020].

12 Arentz M, Yim E, Klaff L, et al. Characteristics and outcomes of 21 critically ill patients with COVID-19 in Washington state. JAMA 2020;323:1612-4.

13 Bhatraju PK, Ghassemieh BJ, Nichols M, et al. Covid-19 in critically ill patients in the Seattle region - case series. $N$ Engl J Med Overseas Ed 2020;382:2012-22.

14 Cummings MJ, Baldwin MR, Abrams D, et al. Epidemiology, clinical course, and outcomes of critically ill adults with COVID-19 in New York City: a prospective cohort study. The Lancet 2020;395:1763-70.

15 Hur K, Price CPE, Gray EL, et al. Factors associated with intubation and prolonged intubation in hospitalized patients with COVID-19. Otolaryngol Head Neck Surg 2020;163:170-8.

16 loannou GN, Locke E, Green P, et al. Risk Factors for Hospitalization, Mechanical Ventilation, or Death Among 10131 US Veterans With SARS-CoV-2 Infection. JAMA Netw Open 2020;3:e2022310-e10.

17 Richardson S, Hirsch JS, Narasimhan M, et al. Presenting characteristics, comorbidities, and outcomes among 5700 patients hospitalized with COVID-19 in the new York City area. JAMA 2020;323:2052-9.

18 Myers LC, Parodi SM, Escobar GJ, et al. Characteristics of hospitalized adults with COVID-19 in an integrated health care system in California. JAMA 2020;323:2195-8.

19 Rizzo S, Chawla D, Zalocusky K. Descriptive epidemiology of 16,780 hospitalized COVID-19 patients in the United States. medRxiv2020.

20 Filardo TD, Khan MR, Krawczyk N, et al. Comorbidity and clinical factors associated with COVID-19 critical illness and mortality at a large public hospital in New York City in the early phase of the pandemic (March-April 2020). PLoS One 2020;15:e0242760.

21 Cavalcanti AB, Zampieri FG, Rosa RG, et al. Hydroxychloroquine with or without azithromycin in mild-to-moderate Covid-19. N Engl J Med 2020;383:2041-52.

22 Abd-Elsalam S, Esmail ES, Khalaf M, et al. Hydroxychloroquine in the treatment of COVID-19: a multicenter randomized controlled study. Am J Trop Med Hyg 2020;103:1635-9.

23 Skipper CP, Pastick KA, Engen NW, et al. Hydroxychloroquine in Nonhospitalized Adults With Early COVID-19 : A Randomized Trial. Ann Intern Med 2020;173:623-31.

24 Hung IF-N, Lung K-C, Tso EY-K, et al. Triple combination of interferon beta-1b, lopinavir-ritonavir, and ribavirin in the treatment of patients admitted to hospital with COVID-19: an open-label, randomised, phase 2 trial. Lancet 2020;395:1695-704.

$25 \mathrm{Li} \mathrm{Y,} \mathrm{Xie} \mathrm{Z,} \mathrm{Lin} \mathrm{W,} \mathrm{et} \mathrm{al.} \mathrm{Efficacy} \mathrm{and} \mathrm{safety} \mathrm{of} \mathrm{Lopinavir/Ritonavir}$ or arbidol in adult patients with Mild/Moderate COVID-19: an exploratory randomized controlled trial. Med 2020;1:105-13.

26 Huang Y-Q, Tang S-Q, Xu X-L, et al. No statistically apparent difference in antiviral effectiveness observed among ribavirin plus interferon-alpha, Lopinavir/Ritonavir plus interferon-alpha, and ribavirin plus Lopinavir/Ritonavir plus interferon-alpha in patients with mild to moderate coronavirus disease 2019: results of a randomized, open-labeled prospective study. Front Pharmacol 2020;11:1071.

27 RECOVERY Collaborative Group. Lopinavir-ritonavir in patients admitted to hospital with COVID-19 (recovery): a randomised, controlled, open-label, platform trial. Lancet 2020;396:1345-52.

28 Beigel JH, Tomashek KM, Dodd LE, et al. Remdesivir for the treatment of Covid-19 - final report. N Engl J Med 2020;383:1813-26.

29 Kalil AC, Patterson TF, Mehta AK, et al. Baricitinib plus Remdesivir for hospitalized adults with Covid-19. N Engl J Med 2021;384:795-807.

30 RECOVERY Collaborative Group, Horby P, Lim WS, et al. Dexamethasone in hospitalized patients with Covid-19. N Engl J Med 2021;384:693-704.

31 Rosas IO, Bräu N, Waters M, et al. Tocilizumab in hospitalized patients with severe Covid-19 pneumonia. N Engl J Med 2021;384:1503-16.

32 Mariette X, Hermine O, Tharaux P-L, et al. Effectiveness of tocilizumab in patients hospitalized with COVID-19: a follow-up of the CORIMUNO-TOCI-1 randomized clinical trial. JAMA Intern Med 2021;181:1241-3.

33 RECOVERY Collaborative Group. Tocilizumab in patients admitted to hospital with COVID-19 (recovery): a randomised, controlled, openlabel, platform trial. Lancet 2021;397:1637-45.

34 Korley FK, Durkalski-Mauldin V, Yeatts SD, et al. Early convalescent plasma for high-risk outpatients with Covid-19. N Engl J Med 2021;385:1951-60.

35 Gattinoni L, Coppola S, Cressoni M, et al. COVID-19 Does Not Lead to a "Typical" Acute Respiratory Distress Syndrome. Am J Respir Crit Care Med 2020;201:1299-300. 
36 Paul V, Patel S, Royse M, et al. Proning in Non-Intubated (pini) in times of COVID-19: case series and a review. J Intensive Care Med 2020;35:818-24.

37 Ioannou GN, O'Hare AM, Berry K, et al. Trends over time in the risk of adverse outcomes among patients with SARS-CoV-2 infection. Clin Infect Dis 2021. doi:10.1093/cid/ciab419. [Epub ahead of print: 11 May 2021].

38 Zhu J, Wei Z, Suryavanshi M, et al. Characteristics and outcomes of hospitalised adults with COVID-19 in a global health research network: a cohort study. BMJ Open 2021;11:e051588.

39 Optum Inc. Optum EHR data, 2021. Available: https://www.optum. com/business/solutions/life-sciences/real-world-data/ehr-data. html2021

40 Optum Inc. Optum COVID-19 data quality services, 2021. Available: https://www.optum.com/content/dam/optum3/optum/en/resources/ brochures/optum-covid-19-data-quality-services.pdf

41 Brooks CG, Brown CA, Cheng A. Abstracts of the 37th International Conference on Pharmacoepidemiology \& Therapeutic Risk Management, Virtual, August 23, 2021. Pharmacoepidemiol Drug Saf 2021;30 Suppl 1:3-439.

42 Medscape, 2020. Available: https://www.medscape.com

43 Medicine. UNLo. ClinicalTrials.gov, 2021. Available: https:// clinicaltrials.gov2021

44 Fine JP, Gray RJ. A proportional hazards model for the Subdistribution of a competing risk. J Am Stat Assoc 1999;94:496-509.

45 Lau B, Cole SR, Gange SJ. Competing risk regression models for epidemiologic data. Am J Epidemiol 2009;170:244-56.

46 Anesi GL, Jablonski J, Harhay MO, et al. Characteristics, outcomes, and trends of patients with COVID-19-Related critical illness at a learning health system in the United States. Ann Intern Med 2021;174:613-21.

47 Asch DA, Sheils NE, Islam MN, et al. Variation in US hospital mortality rates for patients admitted with COVID-19 during the first 6 months of the pandemic. JAMA Intern Med 2021:181:471-8.

48 Rossen LM, Branum AM, Ahmad FB, et al. Excess Deaths Associated with COVID-19, by Age and Race and Ethnicity - United States, January 26-October 3, 2020. MMWR Morb Mortal Wkly Rep 2020;69:1522-7.

49 Finelli L, Gupta V, Petigara T, et al. Mortality among US patients hospitalized with SARS-CoV-2 infection in 2020. JAMA Netw Open 2021;4:e216556.

50 Abeldaño Zuñiga RA, Coca SM, Abeldaño GF, et al. Clinical effectiveness of drugs in hospitalized patients with COVID-19: a systematic review and meta-analysis. Ther Adv Respir Dis 2021;15:17534666211007214.

51 Fan X, Johnson BH, Johnston SS, et al. Evolving treatment patterns for hospitalized COVID-19 patients in the United States in April 2020 July 2020. Int J Gen Med 2021;14:267-71.

52 Brown KA, Jones A, Daneman N, et al. Association between nursing home crowding and COVID-19 infection and mortality in Ontario, Canada. JAMA Intern Med 2021;181:229-36.

53 Cavanaugh AM, Fortier S, Lewis P, et al. COVID-19 Outbreak Associated with a SARS-CoV-2 R.1 Lineage Variant in a Skilled
Nursing Facility After Vaccination Program - Kentucky, March 2021. MMWR Morb Mortal Wkly Rep 2021;70:639-43.

54 Auger KA, Shah SS, Richardson T, et al. Association between statewide school closure and COVID-19 incidence and mortality in the US. JAMA 2020:324:859-70.

55 Bundgaard H, Bundgaard JS, Raaschou-Pedersen DET, et al. Effectiveness of Adding a Mask Recommendation to Other Public Health Measures to Prevent SARS-CoV-2 Infection in Danish Mask Wearers : A Randomized Controlled Trial. Ann Intern Med 2021:174:335-43.

56 Liu Y, Morgenstern C, Kelly J, et al. The impact of nonpharmaceutical interventions on SARS-CoV-2 transmission across 130 countries and territories. BMC Med 2021;19:40.

57 American College of Surgeons. Joint statement: roadmap for Resuming elective surgery after COVID-19 pandemic, 2020. Available: https://www.facs.org/covid-19/clinical-guidance/roadmapelective-surgery2021

58 Tomazini BM, Maia IS, Cavalcanti AB, et al. Effect of dexamethasone on days alive and Ventilator-Free in patients with moderate or severe acute respiratory distress syndrome and COVID-19: the Codex randomized clinical trial. JAMA 2020;324:1307-16.

59 Nuffield Department of Population Health. Low-Cost dexamethasone reduces death by up to one third in hospitalised patients with severe respiratory complications of COVID-19, 2020. Available: https://www. recoverytrial.net/news/low-cost-dexamethasone-reduces-death-byup-to-one-third-in-hospitalised-patients-with-severe-respiratorycomplications-of-covid-19

$60 \mathrm{He}$ J, Guo Y, Mao R, et al. Proportion of asymptomatic coronavirus disease 2019: a systematic review and meta-analysis. J Med Virol 2021:93:820-30.

61 Ora J, Puxeddu E, Cavalli F, et al. Does bronchoscopy help the diagnosis in COVID-19 infection? Eur Respir J 2020;56:2001619.

62 Mak GC, Cheng PK, Lau SS, et al. Evaluation of rapid antigen test for detection of SARS-CoV-2 virus. J Clin Virol 2020;129:104500.

63 İșlek A, Balcı MK. Analysis of factors causing false-negative real-time polymerase chain reaction results in oropharyngeal and nasopharyngeal swabs of patients with COVID-19. Ear Nose Throat $J$ 2021:145561321996621.

64 van Kampen JJA, van de Vijver DAMC, Fraaij PLA, et al. Duration and key determinants of infectious virus shedding in hospitalized patients with coronavirus disease-2019 (COVID-19). Nat Commun 2021;12:267.

65 Zhai P, Ding Y, Wu X, et al. The epidemiology, diagnosis and treatment of COVID-19. Int J Antimicrob Agents 2020;55:105955.

66 Barberi C, Castelnuovo E, Dipasquale A, et al. Bronchoalveolar lavage in suspected COVID-19 cases with a negative nasopharyngeal swab: a retrospective cross-sectional study in a high-impact northern Italy area. Intern Emerg Med 2021;16:1857-64.

67 Polubriaginof FCG, Ryan P, Salmasian $\mathrm{H}$, et al. Challenges with quality of race and ethnicity data in observational databases. J Am Med Inform Assoc 2019;26:730-6. 